\title{
Review on Risk Assessment of Damage of Regional Transmission and Transformation Equipment under Typhoon Disaster
}

\author{
Mao Shuai, Wen Bo, Xu Zhihe \\ School of automation, Wuhan University of Technology, Wuhan, China
}

Keywords: typhoon disaster, risk assessment, modeling

\begin{abstract}
This paper briefly describes the causes and main influences of damage of the power transmission and transformation equipment under typhoon disaster, namely the equipment damage and economic losses in domestic and oversea typhoon disaster caused by the situation, and then it puts forward the power system risk assessment steps. Finally, the probability model and the probability model of all kinds of accidents in the typhoon disaster are set up to calculate the probability of the power system fault. During the typhoon disaster, the power system sometimes appears some situations of large-area inverted towers, broken lines, the phenomenon of tripping, and this can finally lead to long-term large-scale system of power outages. For people's daily production and life there are serious problems, and power companies need under the power grid and power grid repair to bring huge economic losses, so the typhoon disaster on the reliability of the power system is a serious threat. There is a lot of research on the risk assessment of power system, but the risk assessment under the typhoon of the power system is only the initial stage. Therefore, in order to make the emergency design of the transmission line design and the typhoon disaster more scientific and reasonable. To build a more advanced, practical and comprehensive risk assessment model.
\end{abstract}

\section{Introduction}

At present, the world is entering the natural disaster prone period, the El Nino phenomenon lead to increasingly severe weather, typhoon and other chain reaction occurred frequently, caused serious consequences and damage to economic and social life ${ }^{[1-2]}$. The power system outage accidents caused by extreme weather conditions have brought serious harm to the people's production, life and the normal operation of the power system. Extreme weather (typhoons, Blizzard, etc.) is less frequent, but the intensity and destructive force caused by extreme weather can be seen in general. Therefore, the risk assessment of power system for natural disasters is worth attracting the attention of ${ }^{[3-4]}$.

\section{Risk Assessment of Power system under typhoon weather}

\subsection{The cause of the power system failure caused by typhoon}

From the power system component level to system level failure in recent years caused by the internal factors of the power system suffer huge losses, in the process of power system operation due to sudden change of protect misoperation and refused to move and load leads to power system cascading failure phenomenon, resulting in a large area, the loss of power the causes of sudden interruption, the change of load (1) by the end of an important transmission channel fault of power grid, system loss or transmission line fault make power generation capacity or a large amount of lost load. (2) the low controllable climate factors in nature may also cause a sudden increase or decrease in the load. For example, the blackout in Tokyo, Japan, is a system power imbalance due to sudden changes in power generation capacity or load.

\subsection{The influence of typhoon on power system}

In the past few years, China east of Southern China, the southern coastal city suffered typhoon 
damage, the power transmission equipment in power system suffered serious damage, such as power system interrupt pole, pole collapse, loss of power, splitting, the ability of the typhoon landed in Zhejiang in August 12, 2004 14, "Rananim" make the whole substation in Zhejiang province a total of $9220 \mathrm{kV}$ of the power supply, a serious accident of transmission line $500 \mathrm{kV}$ was under the jurisdiction of the Zhejiang power grid tripping over 10 times, $220 \mathrm{kV}$ transmission line trip accident is up to 192 times. In 2005, the "9.26" accident caused widespread blackouts in Hainan power grid.

\subsection{The steps of risk assessment of power system}

Study on risk assessment of power systems, beginning mostly in failure of the event's occurrence stage, in the continuous development, innovation and perfection are now in the risk assessment of power system is the product of the failure probability of the incident and its consequences, this kind of evaluation system can reflect the risk of failure the event of power system. A lot of uncertain factors are brought into the evaluation system to update the input database at any time to make the output data more real-time and more accurate.

Load reduction frequency) and EENS (expected lack of electricity supply). The following is the computing ${ }^{[3,2]}$ of EENS under the typhoon disaster:

$$
E=\int_{0}^{+\infty} p(x) \sum_{i=1}^{n_{p}}\left(T_{i} \sum_{s \in \Omega_{i}} P(s) C(s)\right) d x
$$

Which is the probability of the system in the state; the duration of typhoon impact on the system; the system is in the load cut condition is required; the probability of transmission line failure; regional classification according to the natural environment factors and classification, as expected for the set.

The fourth step is to combine the results obtained from the second and third steps to establish an indicator that can represent the risk of the power system. Risk indicators are varied and can be determined according to specific requirements and actual conditions.2Possibility modeling of damage to power system by typhoon disaster

\section{Possibility modeling of damage to power system by typhoon disaster}

\subsection{Component outage model of power system}

The study on the outage rate, failure frequency and repair time of the outage model can be discussed from two perspectives of macro and micro. Because the failure frequency and outage rate of the component can be analyzed by historical data, it can also be judged by the actual working condition. The former does not consider the status of specific components. The latter is analyzed from the nature, characteristics and damage mechanism of components. In this paper, the damage degree of components is analyzed.

\subsection{Environment dependent model}

In order to calculate the repair time and failure frequency under normal climate and bad weather conditions, we can get the average repair time and average failure frequency based on the past years, and then according to the concept of mathematical expectation, we can get the following conclusions:

$$
\begin{gathered}
f_{t o}=f_{a d} P_{a d}+f_{\text {no }}\left(1-P_{a d}\right) \\
r_{t o}=r_{a d} P_{a d}+r_{n o}\left(1-P_{a d}\right)
\end{gathered}
$$

In fact, failure frequency and adverse weather conditions is the failure frequency of normal climatic conditions, is under adverse weather conditions, the mean time to repair is the average repair time under normal conditions; average failure frequency; probability and adverse weather conditions is the probability of normal climatic conditions; average the repair time of the whole 
period;

\subsection{Outage probability model for overhead transmission lines under large wind conditions}

The line wind load is a group of non-negative random variables, while the line design wind load is also a group of non-negative random variables, and the two all have the dispersive nature. Therefore, the fault probability of overhead transmission lines can be calculated by the stress intensity interference area method under the influence of typhoon.

In case of wind load, the fault probability of transmission line is as follows:

$$
P=1-\int_{0}^{\infty} F\left(w_{d}\right) g\left(w_{d}\right) d\left(w_{d}\right)
$$

Furthermore, the generalized extreme value distribution conforms to the probability distribution of the wind load of the transmission line, that is, the distribution of the wind load of the transmission line:

$$
F\left(w_{x,} \mu_{x,} \sigma_{x,} \xi_{x}\right)=\exp \left\{-\left[1+\xi\left(\frac{w_{x,}-\mu_{x,}}{\sigma_{x}}\right)^{\frac{-1}{\xi_{x}}}\right]\right\}
$$

Secondly, the normal distribution conforms to the probability distribution of the design wind load of the transmission line, that is:

$$
g\left(w_{d}, \mu_{d}, \sigma_{d}\right)=\frac{1}{\sigma_{d} \sqrt{2 \pi}} e^{-\frac{\left(w_{d}-\mu_{d}\right)^{2}}{2 \sigma_{d}^{2}}}
$$

Therefore, we can finally know that the fault probability of the actual overhead transmission line under typhoon disaster is as follows:

$$
P=1-\int_{0}^{\infty} \exp \left\{-\left[1+\xi_{x}\left(\frac{w_{x,}-\mu_{x,}}{\sigma_{x}}\right)^{\frac{-1}{\xi_{x}}}\right]\right\} \frac{1}{\sigma_{d} \sqrt{2 \pi}} e^{-\frac{\left(w_{d}-\mu_{d}\right)^{2}}{2 \sigma_{d}^{2}}} d\left(w_{d}\right)
$$

The parameters of the probability distribution of wind load on overhead transmission lines are: the shape parameter, the location parameter and scale parameter, design parameters of wind load of transmission lines are: standard deviation, circuit design of wind load cell, the mean line load design.

\section{Conclusion}

The evaluation at home and abroad on the typhoon power system is summarized in this paper, which describes the main reasons of the fault occurs in power system under the typhoon, and in accordance with the design standards of the line itself and the environment will be divided into internal and external factors.it helps understand the development status of risk assessment methods and relevant way knowledge by using extreme value theory meanwhile probability theory is used to simulate the probability distribution of all kinds of accidents. The outage model and probability model of various accidents under the typhoon disaster are established. In the end the Monte Carlo method sampling method is also used to determine the probability of the system failure.

\section{References}

[1] Rajendra K.Pachauri, Andy Reisinger.Lenny Bernstein and so on. IPCC_ar4_syr_cn.IPCC, 
2007.Ding Hui, Zhang Jin, Song Yafang. Changes in weather and climate extremes and their connection with global warming [J]. meteorological journal, 2002, 28 (3): 3-7.

[2] Su Sheng, Chen Jinfu, Duan Xianzhong. Interaction between global warming and power system in [J]. power system technology, 2010, 34 (2): 33-40.

[3] Zhang Yufang. Characteristics and statistical distribution of the frequency of typhoon frequency [D]. Shandong: Ocean University of China, 2009.

[4] Xue Yusheng. Overcome the world grid problem Chinese [J]. Chinese information technology magazine, 1997, 2 (11):10-12. 